\section{Ueber einen Fall multipler Primärkrebse} des Dünndarms.

\section{Beitrag zur Frage der multiplen Primärkrebse. \\ Von Dr. Albrecht Freiherrn v. Notthafft, \\ Assistenzarzt am Krankenhause 1. I. Mtinchen.}

Dass verschiedene Carcinome unablaängig von einander an demselben Körper auftreten können, ist eine erwiesene Thatsache Die Arbeiten der letzten Jahre haben in dieser Beziehung vielfach Klarheit geschaffen; namentlich sind hier zu nennen die Schritten von Winiwarter, $\left.{ }^{1}\right) \mathrm{Kauf}$ mann, ${ }^{2}$ ) Michelsohn, ${ }^{3}$ ) Schimmelbusch, ${ }^{4}$ ) Hauser ${ }^{5}$ ) und Bucher. ${ }^{6}$ ) Ein Organ kann zu gleicher Zeit an verschiedenen Stellen carcinomatös erkranken, oder das zweite Primärcarcinom fol gt dem ersten in verschieden langer Zeit nach. In letzterem Falle kann der erste Krebs zu der Zeit, in welcher der zweite auftritt, natürlich oft schon durch das Messer des Chirurgen entfernt worden sein. Je näher aber in dieser Gruppe die beiden Krebse zeitlich beisammen sitzen, um so schwieriger wird die Unterscheidung von einer gewöhnlichen lokalen Krebsmetastase oder einem durch Zurückbleiben eines Geschwulsttheiles, beziehungsweise einer Metastase nach der Operation entstandenen Recidivs. Gleichzeitig multipel auftretende Primärkrebse zeigen eine entschiedene Vorliebe fitlr gewisse Or gane. Vor allem scheint hierzu die Haut disponirt zu sein; aber auch an Zunge, Mundschleimhaut, Magen, Dickdarm, Mamma und Uterus ist mit Sicherheit multiple Bildung von Primärkrebsen gefunden worden, sowohl gleichzeitige, wie nach einander anftretende. Endlich ist eine grosse Reihe von Fällen bekannt, in welchen gleichzeitig oder nach einander an verschiedenen Organen Primärkrebse sich gebildet haben. Mit diesen letzteren haben wir uns in vorliegender Arbeit nicht zu beschäftigen und nehmen daher mit dieser Notiz von ihnen Abschied.

Es ist eine Erfahrungsthatsache, dass die Krebse gern auf einem vorher veränderten Boden entstehen. Meist besteht diese vorausgehende Veränderung in einer typischen Entzündung; ja die letztere ist so häufig, dass in neuester Zeit Ribbert sogar die Hypothese verficht, dass eine Entziundung im Bindegewebe der Carcinombildung voraus gehen müsse, indem die entstehenden Granulationen erst die Epithelien aus ihrem Zusammenhange lostrennen und dadurch zur Krebsbildung Veranlassung geben sollen. (Ich glaube in einer anderen, im Deutschen Archiv für klinische Medicin erschienenen Arbeit den Nachweis geliefert zu haben, dass diese Bindegewebsveränderungen dem Carcinom nicht vorausgehen müs sen, dass sie vielmehr in vielen Fällen secundärer Natur sind.) Vergesellschaftet mit dieser primären Bindegewebsentzlindung oder auch ohne dieselbe rufen senile Veränderungen die Carcinombildung wach. Es ist klar, dass beide Ursachen sich gegenseitig hervorbringen können, besonders die Entzündung einen senilen Charakter des Gewebes. Thiersch selbst verwerthet $z$. B. Narbencarcinome in diesem Sinne; ebenso durfte vielleicht an die Krebsbildung in chronisch entzundetem Gewebe jugendlicher Personen zu erinnern sein.

Es ist nun klar, dass wenn ein Organ in grösserer Ausdehnung von einer zur Carcinombildung prädisponirenden Erkrankung befallen oder in grösserer Ausdehnung senil geworden ist, also verschiedene Stellen in gleichem Sinne verändert sind die Carcinombildung an allen diesen Stellen beginnen kann. Bewiesen wird die Richtigkeit dieser Annahmen durch die multiplen Primärkrebse bei gewissen, meist chronischen Erkrankungen, welche grössere Theile eines Organs einnehmen. Die Paraffin- und Schornsteinfegerkrebse, das seborrhagische Carcinom und der Krebs des Xeroderma pigmentosum sind bekannte Dinge. Bucher citirt in der oben genannten Arbeit noch eine Reihe anderer multipler Primärkrebse: So auf dem Boden von Psoriasis linguae, von Lupus, Lues, nach längerem Arsenikgebrauch und in chronisch entzündeter Magendarmschleimhaut entstanden. Die lokalen Recidive nach Krebsexstirnationen sind in vielen Fällen keine eigentlichen Recidive, sondern vom ersten Carcinom unabhängige Neubildungen, indem an der Stelle des zweiten Carcinoms der Boden erst später die entsprechende Senilität erworben hat, um ebenfalls carcinomatös zu werden. Dass diese senilen Veränderungen häufig nahe beisammen liegen konnen, ist ohne weiteres einzusehen. Oder, wie Bucher sagt: Die multiplen Primärcarcinome sind das genaue Analogon zu Thiersch's regionärem Recidiv, oder dieses ist nur ein spe cieller Theil der grösseren Gruppe. In dieser Weise deute ich mir auch im folgenden meinen eigenen Fall.

Eigener Fall. Als einmal während des Feriencursus, welchen ich in den Osterferien am pathologischen Institut der Universitat Wurzburg gab, Materialmangel eingetreten war, wurde mir von Seiten des anatomischen Instituts in liebensw lirdigster Weise der Cadaver eines an einer croupősen Pneumonie verstorbenen Pfründners zu den Sectionsiibungen zur Verfïgung gestellt. Es ist mir daher leider unmöglich gewesen, eine Krankheitsgeschichte zu bekommen. Die Section, welche äusserst genau ausgefuhrt wurde, ergab folgendes:

Die Schleimhaut des Darmes war in ihrer ganzen Ausdehnung atrophisch und blass, enthielt jedoch nirgends Narben, Pigmentirungen, Ge-

1) Beiträge zur Statistik der Carcinome, 1878.

) Virchow's Archiv Bd. LXXV, 1879.

Michelsohn, Inauguraldissertation, Berlin 1889

Schimmelbusch, Langenbeck's Archiv Bd. XXXIV.

5) Hauser, Das Cylinderepithelium des Magens und Dickdarms. Jena.

6) Bucher, Ziegler's Beiträge Bd. XIV, Heft I.

Da es mir aus äusseren Gründen nicht möglich war, die über diesen Gegenstand handelnde Litteratur mir auch nur annähernd vollzählig zu verschaffen, so entnehme ich die Litteraturangaben grösstentheils aus den hier citirten Werken, welche bei ihrer Genauigkeit dies vollkommen gestatten. schwüre oder andere Veränderungen, welche auf sich noch abspielende oder bereits verflossene entzïndliche Vorgänge hätten schliessen lassen. Ungefähr $10 \mathrm{~cm}$ unterhalb des Endes des Duodenums fand sich gegenüber dem Mesenterialansatz eine Geschwulst von runder, etwas abgeplatteter Form, welche in Gestalt und Grösse einen Stecknadelkopf etwas ubertraf. Die Oberfläche der Geschwulst war glatt; es fehlten sowohl ulceröse Processe, wie auch die normalen Schleimhautfalten nur bis an den Umfang des Knotens heranreichten. Letzterer erhob sich unmittelbar aus einer (mit Ausnahme der Atrophie) ganz normalen Schleimhaut. A uf dem Durchschnitt zeigte das Geschwulstchen eine mattweisse Farbe. Die Consistenz war eine ziemlich harte. Etwa $10 \mathrm{~cm}$ unterhalb des ersten Knotens fand sich ein zweiter, und etwa $5 \mathrm{~cm}$ unterhalb des letzteren ein drittes Knötchen. Sie glichen in Form, Farbe, Consistenz, überhaupt nach jeder Richtung hin vollkommen dem ersten; nur in der Grösse bestand ein Unterschied, indem das mittlere Knötchen etwa Erbsen-, das dritte Kirschkerngrösse besass; der Höhendurchmesser war bei jenen Geschwullsten ungefähr der dritte Theil des Breitendurchmessers. Die drei Knötchen sahen so wenig wie Carcinome aus, dass ich die Diagnose des die Obduction ausfithrenden Studenten "Polyposis intestinalis" nicht zu corrigiren mir getraute. Trotzdem wurde die weitere Obduction in peinlichst genauer Weise vorgenommen, aber nirgends fand sich irgendwo im Körper irgend eine durch eine Neubildung hervorgerufene Veränderung. Dagegen wurden neben einer alten Gehirnhämorrhagie und einer croupösen Pneumonie noch die verschiedensten Altersveränderungen in allen Organen beobachtet.

Das erkrankte Darmstack wurde sammt dem dazu gehörigen Mesen terium herausgenommen, die drei Knötchen gehärtet, eingebettet und mit dem Mikrotom in Schnitte zerlegt. Ausserdem wurden auch ein paar Sttickchen der zwischen den Knötchen gelegenen Dünndarmtheile, des Mesenteriums und einige benachbarte Lymphdrüsen genau untersucht.

Mikroskopisch liess sich folgendes nachweisen: Der Darm zeigt, ab. gesehen von geringer Atroplie der Schleimhaut, nirgends irgend welche Veränderungen, also auch keine entzindlichen.

Oberstes Knötchen: Auf einer etwas verschmälerten Muskelschicht rhebt siclı von der Submucosa aus eine mächtige Bindegewebswucherung, welche sich stark uber das Niveau der angrenzenden Schleimhaut erhebt. Das Bindegewebe zeigt einen durchaus scirrhösen Charakter; viele straffe Fasern, wenig Kerne, wenig Gefässe. An vielen Stellen findet sich kleinzellige Infiltration. Kleinzellig infiltrirt sind auch die benachbarten Schleim. hautbezirke. Die Drüsen und Zotten klettern noch etwas auf den Tumor hinauf, bald aber hören sie vollständig auf. Die letzten Drüsen zeigen bereits richtige krebsige Veränderungen, Infarcirung ihres Lumens mit massenhaft gebildeten Epithelien und seitliche Sprossung von an Drüsen erinnernden Epithelschläuchen. Die Oberfläche des Knötchens ist glatt und nackt; ein ursprünglich vielleicht vorhandenes Epithel ist wohl infolge postmortaler Einwirkung (ich bekam die Leiche erst einige Tage nach eingetretenem Tode) nicht mehr zu sehen. Innerhalb der Geschwulst fehlen Drüsen vollständio. Dagegen findet sich dieselbe vollständig durchsetzt von drisenähnlichen Gebilden, das sind Züge und Haufen von Epithelzellen von geringer Grösse, (mit Hämatoxylin) dunkel sich färbendem Protoplasma und noch dunkler sich färbendem Kerne. Ihre Form wechselt ausserordentlich; ebenso die des Kernes. Meist liegen in den Zellzügen drei oder zwei Epithelien neben einander, in den Zellhaufen entsprechend mehr. Regressive Veränderungen sind an diesen Epithelien nicht zu sehen doch muss ich bemerken, dass, weil die Leiche nicht mehr frisch, also die Fäulnissvorgänge schon ziemlich weit vorgescliritten waren, die Färbung nicht mehr genügend gut gelang, um solche mikroskopischen Feinheiten mit entsprechender Schärfe hervortreten zu lassen. Die Haufen liegen mehr an der Oberfläche der Geschwulst, die Zïge mehr in den anderen Theilen. Vielfach sind natürlich die Zellstränge auf dem Querschnitte sichtbar. Die Krebsgebilde finden sich immer in den Spalträumen des Bindegewebes; manchmal scheinen solche Räume mit einem Endothel ausgekleidet $\mathrm{zu}$ sein. - Die Muscularis ist, wie gesagt, leicht verschmälert; die Krebszüge sind noch nicht über die Submucosa hinaus vorgedrungen.

Mittleres Knotchen: Die Muscularis ist hier noch mehr verschmälert, sonst die Verhältnisse wie bei dem vorher beschriebenen Knötchen.

Unterstes Knötchen: Das Gleiche wie an den beiden anderen Die krebsioe Infiltration ist hier jedoch in 1-2-3 Enithelzellen in dicken Zügen zwischen die Bündel der Ringmuskulatur und zwischen die beiden Muskelstrata bereits hineingerathen. An diesen Stellen findet sich etwas kleinzellige Infiltration.

Ebensowenig wie in den untersuchten Schleimhautstïckchen konnte mesenterium und den Lymphdrüsen Carcinom mikroskopisch nachgewiesen werden.

Das Resultat dieser Untersuchungen liess die Diagnose „Schleimhautpolypen" nicht mehr aufrechterhalten, sondern zwang zu der Annahme, dass man es hier mit drei Carcinomen des Darmes $z u$ thun hatte, und zwar primären Drisencarcinomen, nicht etwa carcinomatös veränderten Schleimhautpolypen.

Epikrise: Der geschilderte Fall lässt die Frage aufwerfen, auf welche Weise die drei Carcinomknötchen entstanden sind. Sind sie von einander unabhängig oder abhängig zur Entwickelung gelangt, haben sie eine gemeinsame Ursache, oder lässt sich eine solche nicht nachweisen? Zunächst sind auf jeden Fall drei Möglichkeiten zu berücksichtigen:

1. Zwei der Carcinome sind Metastasen des dritten.

2. Von einem der Carcinome wurden in den Darm specifisch ,infectiosse" Stoffe abgesondert, welche an zwei anderen Darmstellen durch "Infection" secundäre (aber nicht metastatische) Knoten erzeugten. standen.

Die drei Carcinome sind vollständig unabhängig von einander ent- 
Ad 1. Zwei der Krebse sind Metastasen des dritten. In diesem Falle müssten also an einem der Knötchen die Zeichen einer primären Drüsen- und Epithelwucherung sich finden, an den anderen mîssten dieselben unbetheiligt sein, das Carcinom müsste sozusagen subepithelia liegen; anerkanntermaassen finden sich die Darmmetastasen fast immer in der Submucosa. Auf jeden Fall darf sich keine Entstehung aus dem Epithel und den Drüsen der Umgebung dieses secundären Krebses finden lassen. Werden nun die thatsächlich vorliegenden Verhältnisse diesen Forderungen gerecht? Die Schilderung des mikroskopischen Befundes hat gezeigt, dass sich die drei Knötchen nur durch ihre Grösse und ihr Alter unterschieden, dass sich aber an jedem die Betheiligung der lokalen epitheltragenden Theile nachweisen liess. An jedem der Knötchen zeigte sich eine krebsige Umwandlung der obersten Schleimhautschichten, von welcher dann infiltrative Züge durch die Submucosa gegen die Muscularis zogen. Die stärksten, massigsten carcinomatösen Veräuderungen fanden sich immer an der Grenze gegen das Darmlumen, die schwächsten in der Nähe der Muscularis. Bei genauer Untersuchung vieler Schnitte liessen sich in den Randpartieen eines jeden Knötchens Stellen nachweisen, wo sich Langerhans'sche Bilder, d. h. die fortschreitende Neubildung von Krebs aus alten Drüsen, fanden. Kurz! eine Uebereinstimmung der Geschwülste unter einander bis in's kleinste, und eine evidente Abhängigkeit der Neubildung von dem Epithel des Ortes, an welchem sie entstanden war. Ist man aus diesen Gründen schon berechtigt, die Beweise für eine dreifache primäre Carcinombildung als erbracht anzusehen, so wird man in dieser Ansicht bestärkt, wenn man das Folgende bertucksichtigen will: Carcinonimetastasen in den Darm sind überhaupt etwas sehr Seltenes. Sie können entstehen durch eine Weiterverschleppung der Krebspartike in die benachbarten Darmlymphgefässe oder auf retrogradem Wege aus den Lymphgefässen des Mesenteriums. Für erstere Möglichkeit liegen die Knoten doch etwas zu weit auseinander und ist als gegensprechend zu betrachten, dass der Darm zwischen den einzelnen Knötchen nirgends eine krebsige Infiltration seiner Lymphgefässe, nirgends eine auch nur mikroskopisch nachweisbare Metastase zeigte. (Gegenüber dem Einwand, dass ich ja nicht Serienschnitte durch die zwischen den Knoten gelegenen Darmstrecken angelegt habe, dass mir also krebsige Veränderungen entgangen sein können, welche sich eventuell in anderen [nicht angelegten] Schnitten gefunden hätten, glaube ich die Ansicht aussprechen zu dïrfen, dass dieser Einwand doch an einer sehr schwachen Begrtundung leidet, indem so entwickelte Krebsmetastasen doch zu der Erwartung berechtigen, dass eine ausgedehntere submucose Lymphgefässerkrankung stattgefunden hätte, welche sich in den wenigen zur Untersuchung gelangten Schnitten hätte auffinden lassen muissen; eine ein- oder höchstens zweimal und dann nie wieder, weder in den Darm noch sonstwohin erfolgte Ver schleppung von Krebszellen zur Erklärung der zwei "Metastasen" annehmen müssen, hiesse ja mit den unwahrscheinlichsten Unwahrscheinlichkeiten arbeiten.)

Eine Metastasirung auf retrogradem Wege lässt sich kaum als möglich annehmen, wenn man bedenkt, dass makroskopisch und mikroskopisch die Lymphdrüsen únd Lymphgefässe des Mesenteriums vollkommen gesund waren. Mit der Annahme einer metastatischen Erkrankung lässt sich überhaupt sehr schwer der Umstand vereinigen, dass sich nirgends im Körper, ausser an den drei genannten Darmstellen, irgend welche carcinomatösen Veränderungen nachweisen liessen, und dies, obwohl ich versichern kann, dass die Section der Leiche, welche ich ja in $\mathrm{Ob}$ ductionscurse habe ausfïhren lassen, in der gewissenhaftesten Weise geschehen ist.

Ad 2. Von einem der Carcinome wurden in den Darm specifische "infectiöse" Stoffe abgesondert, welche an zwe anderen Darmstellen durch Infection (nicht durch Metastase) secundäre Knoten erzeugten. Mit Recht betont Bucher, dass die Hypothese von der Implantation von Carcinom auf epithelbedeckte Flachen - Lungenalveolen, Beck's Fall von Magencarcinon und vielleicht Ovarien abgerechnet - noch nichts weniger als begrundet, sogar für nicht wenige Fälle unwahrscheinlich ist. - Diejenigen, welche an der Idee einer Implantation des Krebses von epithelbedeckter Fläche her festhalten. übersehen vollständig die enormen Schwierigkeiten, welche hierbei in Frage kommen. Wenn schon der Versuch der künstlichen Implantation von Krebs so ausserordentlich selten gelingt, wo doch vom Experimentator kunstlich die gunstigsten Fortpflanzungsbedingungen geschaffen wor den sind, wie soll man da annehmen, dass nur der Theorie zu Liebe hier eine Krebsimplantation auf epitheltragende Schleimhaut, beziehungsweise Epidermis (boi der äusseren Haut) stattfinden köune? Man versuche es nur einmal, Thiersch'sche Transplantationen auf das intacte Oberflächenepithel aufzutragen, und man wird Fiasco machen! Aber gesetzt auch, dass Schleimhaut, beziehungsweise äussere Haut schwache Stellen besässen, d. h. infolge von 'Traumen oder Geschwüren irgendwo epithellos geworden wären, so ist wenigstens der Schleimïberzug der Darmschleimhaut für die Krebszelle jedenfalls noch ein undurchdringliches Hinderniss. Nicht nur der Inoculotionsboden ist ein ungünstiger, das Krebssperminium selbst sehr tief unter dem Versuchsmaterial. Die Krebszellen, welche von der Oberfläche der ersten Knoten abgelöst werden, sind die ältesten des betreffenden Knotens und daher auch am wenigsten lebens-, beziehungsweise infectionsfähig; in der Regel werden sie gerade deshalb abgestossen, weil sie nicht mehr lebensfähig sind. Es ist forner schwer verständlich, dass solche abgelösten Zellen durch die Darmsäfte, durch Fäulnissvorgänge und Bacterien nicht geschädigt, ja sogar zerstört werden, warum nich auch sie nun dem Verdauungsprocesse unterworfen werden sollen. Die Schädigungen und Schwierigkeiton, welche die Krebszelle verhindern, an der äusseren Haut Inoculationskrebse zu erzeugen, sind natürlich andere als im Darm, aber kaum minder in's Gewicht fallonde. Dass Zellen, welche sich von Carcinom geschw üren ablosen, in keiner Weise als implantationsfähig angesehen werden können, ist selbstverständlich. Der von
Erbse ${ }^{1}$ ) geschilderte Fall, in welchem ein Oesophaguscarcinom in den arynx durchgebrochen war und wo sich dann (wic er annimmt, durch Aspiration verschluckter Krebspartikel) Lungencarcinom entwickelt hat, unterscheidet sich von den ubrigen bekannten als Implantationskrebse gedeuteten Fällen vor allem dadurch, dass hier die jüngsten Krebszellen, die eben durchgebrochenen, zur Implantation gefithrt liatten und dass in den Lungenalveolen die Dünnheit des Epithels und der Fortfall aller Schädlichkeiten, welche sich im Darme und an der äusseren Haut finden. eine Krebsinoculation viel leichter zulassen. (Diese Ueberlegung führt bereits Bucher l. c. an.) Eine Beobachtung von $\mathrm{Beck}^{2}$ ), wo in einem Falle von grossem ringförmigem carcinomatösem Geschwtï in der Speiseröhre mit Durchbruch in den linken Bronchus u. a. auch ein kleineres Knötchen über der Cardia gefunden wurde, lässt allerdings die Möglichkeit einer Krebsinoculation an letzterer Stelle nicht ausschliessen. Auch für die Ovarien sind vielleicht derartige Beobachtungen gesammelt. Immerhin ist daran festzuhalten, dass eine Krebsentwickelung durch natürliche Implantation, wenn sie überhaupt vorkommt, jedenfalls ausserordentlich selten ist und sich bis heute noch nicht hat beweisen lassen. Die meisten bisher veröffentlichten Fälle halten vor einer eingehenden Kritik nicht Stand, sie lassen sich recht gut entweder als Doppelbildungen oder als auf dem Wege der retrograden Lymphgefässmetastase entstanden erklären. Auch jene Fälle, wo von zwei sich berührenden Korperstellen nach einander jede carcinomatös entartete, braucht man nicht als Beweis des Zustandekommens förmlicher Abklatschcarcinome zu erklären, sondern vertragen dieselben Erklärungsversuche, wie die anderen Doppelkrebse; ausserdem hindert nichts anzunehmen, dass in den Fällen, in welchen zuerst ein Carcinom an der einen und erst später ein solches auf der anderen Lippe, beziehungsweise Muttermundslippe sich zeigte, oder in welchen an derjenigen Stelle des einen Oberschenkels, wo eine Krebsgeschwulst des anderen Oberschenkels hindrückte, in der Folge ebenfalls ein Krebs entstand, das erste Carcinom, sei es durch mechanischen Druck, sei es durch chemische Irritation das zweite erzeugt hat, wie eben uberhaupt Carcinome infolge mechanischer oder chemischer Insulte entstehen.

Die Erfahrungen und theoretischen Erwägungen sind also für die Deutung unserer Präparate als durch Krebsimplantation entstanden, schon gar nicht günstig. Aber auch das im Vorhergehenden geschilderte Material selbst, die Art und Weise der Darmwucherungen spricht gegen eine Krebsinoculation. Denn man musste doch, um letztere Möglichkeit vertheidigen zu können, verlangen, dass das oberste Knötchen das älteste, das unterste das kleinste wäle, entsprechend der späteren Entstehung des letzteren, indem mit den Bewegungen des Darminhaltes das Krebsseminiunı von oben nach unten geschoben worden wäre. Gerade das Umgekehrte ist jedoch der Fall. Das oberste Knötchen war das kleinste, das unterste das grosste. - Wenn ferner das Zustandekomnien eines einzigen Krebses durch Implantation schon ausserordentlich selten sein muss (wenn sie überhaupt vorkommt), so wird eine derartige Bildung von $\mathrm{z}$ wei solchen Krebsen doch höchst unglaublich, während sich drei Carcinome a priori ja recht gut als Metastasen eines von ihnen, oder als Dreifachbildungen auf einem gemeinsam gleichartig veränderten Untergrund erklären.

Da, wie schon Bucher und Schimmelbusch hervorgehoben haben, die mykotische Theorie der Krebsentwickelung keine Stütze durch die Multiplicität erhält, so können wir hier auf eine Berïcksichtigung derselben verzichten.

Ad. 3. Die Carcinome sind vollständig unabhängig von einander entstanden. Nach Ausschluss der beiden anderen Möglichkeiten müssen wir die dritte zunächst als wahrscheinlich hinstellen. Bei genauerem Zusehen dürfte jedoch diese Wahrscheinlichkeit so erstarken, dass kaum ein Zweifel mehr darüber walten kann, dass diese Annahme die allein berechtigte ist. Die Darmschleimhaut zeigte zwar nirgends die nächste Umgebung der: Carcinome ausgenommen - die Spuren acuter oder chronischer Entztindung, dagegen war eine Atrophie derselben, eine Verkarzung und Verschmälerung der Zotten und Drüsen ziemlich stark ausgesprochen. Die Darmgewebe waren also in grösserer Ausdelınung senile Veränderungen eingegangen; eine gemeinsame Ursache für die Entstehung sänmtlicher drei Krebse war soinit gegeben. Jedes der drei Knotchen ontsprach sodann den Forderungen, welche nian an ein Primärcarcinom stellen kann: Jede Geschwulst sass in der Schleimhaut selbst, nahm ihren Ausgang von den Driisen des Ortes, war am stärkstell entwickelt gegen das Darnilumen, am schwächsten und nuit Ausläufern in der Submucosa. Das unterste Knötchen ist waluscheinlich das älteste, an den beiden anderen Stellen hat vielleicht der Darm erst später die entsprechende "Senilität" erreicht.

Jedem einzelnen Knoten entspracl eine leftige reactive Eutzündung und Neubildung des Bindegewebes, welche ganz scharf auf die nächste Umgebung des Carcinoms und letzteres selbst beschränkt blieb; dieser Umstand, dass nur da, wo der Krebs sass, eine Bindegewebsvermehrung vorhanden war, welche sicl in ihrer Form ganz streng an die Formen und Grenzen des Carcinoms hielt, scheint mir nicht sehr für die Ribbert'sche Ansicht zu sprechen, dass die Krebse sich infolge einer primären Bindegewebserkrankung ontwickeln würden. In diesem Falle wenigstens scheinen die Bindegewebsveränderungen sichel das secundäre $\mathrm{zu}$ sein.

Multiple Krebse des Intestinaltractus sind bereits von Klebs, Erbse, Beck, Kraske, Hauser, Lucke, v. Bercrmann u. a. (siehe die genauen Litteraturangaben bei Bucher und Schimmelbusch) beschrieben worden. Multiple Carcinome des Dunndarms, speciell seiner oberen Theile, des Jejunums, sind jedenfalls ausserordentlich selten. Mir ist wenigstens nur der Fall von $\mathrm{Hjelt}$ bekannt geworden, in welchem sich auf ein ulcerirtes Duodenalcalcinom hin Ileunt- und Colonkrebse ent-

1) Erbse, Inauguraldissertation, Halle 1884.

2) Beck, Prager Zeitschrift für Heilkunde 1884, H. 6. 
wickelten. Primärkrebse dürten die letzteren wohl kaum gewesen sein. Auf Grund solcher Erwïgungen wurde der Fall als Beitrag zur Casuistik veröffentlicht. ${ }^{1}$ ) 